University of South Carolina

Scholar Commons

1990

\title{
Rheology of Aqueous Suspensions of Polystyrene Latex Stabilized by Grafted Poly(Ethylene Oxide)
}

Harry J. Ploehn

University of South Carolina - Columbia, ploehn@cec.sc.edu

J. W. Goodwin

Follow this and additional works at: https://scholarcommons.sc.edu/eche_facpub

Part of the Materials Chemistry Commons

\section{Publication Info}

Published in Faraday Discussions of the Chemical Society, Volume 90, 1990, pages 77-90.

(C) Faraday Discussion of the Chemical Society 1990, Royal Society of Chemistry

Ploehn, H. J. \& Goodwin, J. W. (1990). Rheology of aqueous suspensions of polystyrene latex stabilized by grafted poly(ethylene oxide). Faraday Discussions of the Chemical Society, 90, 77-90.

http://dx.doi.org/10.1039/DC9909000077

This Article is brought to you by the Chemical Engineering, Department of at Scholar Commons. It has been accepted for inclusion in Faculty Publications by an authorized administrator of Scholar Commons. For more information, please contact digres@mailbox.sc.edu. 


\title{
Rheology of Aqueous Suspensions of Polystyrene Latex stabilized by Grafted Poly(Ethylene Oxide)
}

\author{
Harry J. Ploehn \\ Department of Chemical Engineering, Texas A\&M University, College Station, \\ Texas 77843-3122, USA
}

\author{
James W. Goodwin \\ Department of Physical Chemistry, University of Bristol, Cantock's Close, Bristol BS8 1TS
}

\begin{abstract}
A water-soluble carbodiimide has been used to end-graft aminated poly(ethylene oxide) (PEO) chemically onto colloidal polystyrene particles. Two particle sizes (115 and $347 \mathrm{~nm}$ diameter) and two PEO molecular weights ( 112000 and $615000 \mathrm{~g} \mathrm{~mol}^{-1}$ ) were combined to give suspensions with four different ratios of polymer layer thickness to particle radius. Electrophoresis demonstrated that the PEO was grafted, not just adsorbed. Dynamic light scattering showed that the adsorbed and grafted layers had similar structures and that non-ionic surfactant perturbed the PEO configurations. Steady shear and oscillatory rheometry indicated that long-ranged polymeric forces between particles governed the variation of viscosity and storage modulus with applied stress and PS volume fraction. Hard-sphere and effective hard-sphere scaling helps rationalize the rheological behaviour in terms of the variation of the polymeric force among the different suspensions and hydrodynamic deformation of the polymer layers.
\end{abstract}

Polymers are widely utilized to modify the properties of colloidal suspensions. The use of polymers to stabilize suspensions has been well documented, ${ }^{1,2}$ and polymeric flocculation has been employed in several industries. ${ }^{3}$ Invariably, the polymeric additive also alters the bulk theology of the colloidal suspension through at least one of three mechanisms: the polymer's influence on the state of aggregation of the suspension, more subtle alteration of the suspension microstructure via the polymer-mediated interparticle force, direct modification of the rheological properties of the suspending fluid. In some cases, the polymeric modifier is added primarily to control the bulk rheology. When the polymer is soluble in the suspending fluid, its effect on the system can be classified according to the polymer's interaction with the suspended particles: depletion, adsorption and grafting modes have been studied extensively. ${ }^{4}$

Physical circumstances often dictate the role of the polymer and its mode of use in a given situation. In aqueous suspensions, electrostatic forces confer stability, so the polymer's role has most often been that of flocculant; through the adsorption mode, the polymer can promote aggregation by partially neutralizing the stabilizing charges and by forming bridge configurations between particles. In organic media, the dominance of the van der Waals attraction over electrostatic repulsion encourages the use of polymers as stabilizers; both adsorption and grafting modes have been employed. In some cases grafted stabilizers have an advantage: if the polymer has no affinity for the surface (relative to that of the solvent), then the possibility of bridging can be avoided.

These generalizations, though, are becoming less valid as industrial needs and academic interests change. In particular, economic and environmental considerations favour less reliance on organic solvents and greater use of aqueous media. It would be 
helpful to be able to use established techniques for polymeric stabilization in aqueous systems. Progress in this area requires further development of polymer stabilizer technology, especially with regard to: (i) understanding the structure and behaviour of polymer layers, both in organic and aqueous colloidal suspensions; (ii) characterizing the effect of the polymer on the suspension microstructure and bulk properties; and (iii) identifying and understanding effects peculiar to aqueous systems, such as polymersurfactant interactions, polymer electrostatic coupling, and so on.

The rheology of polymer-stabilized suspensions depends on the Brownian motion of the particles, hydrodynamic interactions of the particles with the fluid and each other, and the total interparticle force. Early efforts focused on low molecular weight stabilizers of two types: block copolymers with an insoluble block, which absorbed on the growing particles and stabilized them during polymerization; ${ }^{5,6}$ and functionalized homopolymer, which was chemically grafted onto the particles in a post-polymerization step. ${ }^{7,8}$ The resultant polymer layers had high graft densities and low thicknesses, which produced a steep interparticle force that could be approximated by an effective hard-sphere interaction. Dynamic light scattering (DLS) measurements ${ }^{9}$ indicated that silica particles stabilized by octadecyl alcohol ${ }^{7}$ in cyclohexane interacted as hard spheres. Rheological measurements ${ }^{10}$ agreed with theoretical predictions for dilute hard spheres $^{11}$ and, at higher volume fractions, could be rationalized through 'Brownian hard-sphere scaling. ${ }^{12}$

For poly(methylmethacrylate) (PMMA) particles stabilized by poly(12-hydroxystearic acid), the stabilizer layer thickness could be distinguished from the particle radius. ${ }^{13}$ When the ratio of the thickness to the radius was small, the rheological data ${ }^{13,14}$ displayed Brownian hard-sphere behaviour, provided the effective particle volume fraction was calculated from the particle radius plus the stabilizer thickness. However, the high- and low-shear limiting viscosities differed somewhat from those found in the silica suspensions. Hard-sphere behaviour was also observed for poly(vinyl chloride) plastisols in alkyl phthalate plasticizers ${ }^{15}$ and PMMA suspensions stabilized by poly(dimethylsiloxane) in low molecular weight silicone oils. ${ }^{16}$

Deviations from hard-sphere scaling appeared ${ }^{13}$ when the thickness:radius ratio became large, implying that deformation of the polymeric stabilizer was responsible. A number of studies ${ }^{13,15-23}$ have characterized 'soft' long-range polymeric interactions in suspensions through rheological measurements. The details of the interaction, and hence the rheological behaviour, depended not only on the chemistry and molecular weight of the stabilizer and the thickness: radius ratio, but also on the composition of the medium $^{15,18}$ its structure, ${ }^{16}$ and the temperature. ${ }^{19}$ Few studies have examined the rheology of suspensions stabilized by high molecular weight polymer ${ }^{19,20,22}$ or stabilized suspensions in aqueous media. ${ }^{21,23}$

The objective of this work is to characterize polymer-mediated interparticle interactions in aqueous suspensions of polystyrene (PS) stabilized by high molecular weight poly(ethylene oxide) (PEO) through electrophoresis, dynamic light scattering, and rheological measurements. Utilizing a technique originally developed for grafting biochemical ligands onto colloidal substrates for agglutination tests, ${ }^{24}$ the PEO was chemically end-grafted onto active sites on the surfaces of the PS particles in water. Electrophoresis measurements demonstrated that the PEO was anchored to the PS, not just adsorbed. Four samples, each having a different thickness: radius ratio, were prepared from two PEO molecular weights and two PS particle sizes. Dynamic light scattering and capillary viscometry measurements gave the hydrodynamic radius of the coated particles and confirmed that the PEO layer structure was typical of that for adsorbed polymers. ${ }^{25,26}$ Steady shear and oscillatory rheometry provided data that reflected the influence of the polymer-mediated interparticle force on the suspension microstructure and bulk rheology; differences between suspensions with varying degrees of particle softness were apparent. 


\section{Experimental}

\section{Materials}

Several suspensions of PS particles were prepared using standard emulsion polymerization techniques ${ }^{27}$ and published recipes. ${ }^{28}$ The polymerizations were initiated with 4,4'-azobis-4-cyanopentanoic acid (Wako Chemicals $\mathrm{GmbH}$ ) and produced particles stabilized by surface carboxyl groups. Suspension PS0 was prepared without added surfactant; suspension PS1 resulted from the seeded growth of $31 \mathrm{~nm}$ PS particles, also without added surfactant; and suspension PS2 was prepared with surfactant, 12dodecanoic acid. The suspensions were dialysed for more than a month against frequent changes of twice-distilled water. TEM gave particle diameters of 375,115 , and $347 \mathrm{~nm}$ for PS0, PS1, and PS2, respectively; a diffraction grating calibrated the measurements. Optical sizing of photographic images ( $>500$ per sample) yielded standard deviations in diameter of 4,5 and $2 \%$ for PS0, PS1, and PS2, respectively. Conductometric titration of PS0 resulted in a surface charge density of $c a .0 .5 \mu \mathrm{C} \mathrm{cm}$.

Three PEO samples were used as received: PEO0, a commercial grade sample with $M_{\mathrm{w}} \approx 1.0 \times 10^{5} \mathrm{~g} \mathrm{~mol}^{-1}$ and $M_{\mathrm{w}} / M_{\mathrm{n}} \approx 6.6 ;$ PEO1, a Polymer Labs standard with $M_{\mathrm{w}}=$ $1.12 \times 10^{5} \mathrm{~g} \mathrm{~mol}^{-1}$ and $M_{\mathrm{w}} / M_{\mathrm{n}}=1.03 ;$ PEO2, another Polymer Labs standard with $M_{\mathrm{w}}=$ $6.15 \times 10^{5} \mathrm{~g} \mathrm{~mol}^{-1}$ and $M_{\mathrm{w}} / M_{\mathrm{n}}=1.10$.

All other reagents were $\mathrm{BDH}$ AnalaR grade. A water-soluble carbodiimide (WSC), 1-ethyl-3-(3-dimethylaminopropyl) carbodiimide (Fluka), served as the linking agent in the grafting reaction. Monodisperse non-ionic surfactant hexaethylene glycol mono-ndodecyl ether (Nikko Chemicals), henceforth denoted as $\mathrm{C} 12 \mathrm{E} 6$, was used as received.

\section{Grafting Reactions}

To prepare the PEO for grafting, its hydroxyl end group was converted to an amine group. PEO was dissolved in warm toluene at concentrations of $0.5-5.0 \times 10^{-2} \mathrm{~g} \mathrm{~cm}^{-3}$ (depending on the molecular weight). A ten-fold molar excess of toluene-4-sulphonyl chloride and $0.5 \mathrm{~cm}^{3}$ pyridine were added, thereby replacing the PEO hydroxyl end groups with tosylate leaving groups and precipitating insoluble pyridinium hydrochloride. Bubbling ammonia gas through the warm $(303 \mathrm{~K})$ stirred solution for 3-5 $\mathrm{h}$ gave end-aminated PEO (APEO). Most of the toluene was then evaporated off under vacuum. The APEO was dissolved in dioxane, freeze-dried to remove organic solvents, and redissolved in water at $0.5-5 \%$ by weight.

Although the mechanism is rather complicated, ${ }^{29}$ the overall WSC grafting reaction is straightforward: WSC and APEO were added to the suspensions of carboxylated PS particles, giving PS particles with APEO grafted through amide linkages plus a diurea byproduct. The APEO dosage, estimated from equilibrium adsorption data, ${ }^{26}$ was gauged to provide an adsorbed amount on the plateau of the adsorption isotherm; for $6.6 \times 10^{5} \mathrm{~g} \mathrm{~mol}^{-1} \mathrm{PEO}$, a solution concentration of $2.0 \times 10^{-4} \mathrm{~g} \mathrm{~cm}^{-3} \mathrm{gave}^{26}$ an adsorbed amount of $1.42 \times 10^{-7} \mathrm{~g} \mathrm{~cm}^{-2}$. These values were used in conjunction with the particle diameter and volume fraction to compute the amount of APEO to be added to each suspension. To minimize bridging flocculation, the particles were added to stirred, diluted APEO solution so that the final particle volume fraction was 0.015 . The suspensions were polymerically stabilized at this point. WSC was then added to give one molecule per $10 \mathrm{~nm}^{2}$ of particle surface, and the mixture was stirred overnight at $303 \mathrm{~K}$. This procedure was followed for the following particle-polymer pairs: PS1-APEO1, PS1-APEO2, PS2-APEO1 and PS2-APEO2. PS2-APEO2 was grafted at $273 \mathrm{~K}$.

A slightly different procedure was followed for PS0-APEO0. N-hydroxybenzotriazole was grafted onto PS0 to form an intermediate active ester. In principle, the stability of this ester allows cleaning of the suspension, removing excess WSC which might otherwise damage the molecule to be grafted. 'The benzotriazole ester is a good 
leaving group, so the amide linkage is easily formed when APEO is added. Here, APEO0 was mixed with uncleaned intermediate and stirred at $273 \mathrm{~K}$.

\section{Sample Preparation}

Samples for electrophoretic mobility and DLS measurements were diluted by a factor of 5000 with filtered $10^{-3} \mathrm{~mol} \mathrm{dm}^{-3}$ sodium acetate buffer solution at $\mathrm{pH} 6.3$; the $\mathrm{pH}$ was adjusted to other values through addition of sulphamic acid or sodium hydroxide. Other samples were diluted into buffer also containing $4.0 \times 10^{-4} \mathrm{~mol} \mathrm{dm}^{-3} \mathrm{C} 12 \mathrm{E} 6$.

Suspensions stabilized by the high molecular weight APEO, although not flocculated after centrifugation, could not easily be redispersed; sonication was not used for fear of degrading the APEO. Consequently, all samples for rheological measurements were treated with sufficient C12E6 surfactant to give monolayer coverage of the particles in equilibrium with $4 \times 10^{-4} \mathrm{~mol} \mathrm{dm}{ }^{-3} \mathrm{C} 12 \mathrm{E} 6$ in solution. ${ }^{30}$ Subsequent low-speed centrifugation removed any flocculated material. The samples were cleaned and concentrated through multiple cycles of centrifugation and redispersion into $10^{-3} \mathrm{~mol} \mathrm{dm}^{-3}$ sodium acetate and $4 \times 10^{-4} \mathrm{~mol} \mathrm{dm}^{-3} \mathrm{C} 12 \mathrm{E} 6$. The buffer ionic strength was high enough to make the electrostatic double-layer thickness less than the APEO layer thickness, yet low enough to minimize perturbations of the APEO configurations. After centrifugation, the samples redispersed very easily. Particle volume fractions were determined by dry weight analysis, neglecting the weight of the APEO and taking $1.05 \mathrm{~g} \mathrm{~cm}^{-3}$ as the density of PS.

\section{Characterization Methods}

Particle electrophoretic mobility was measured with a Pen Kem System 3000 Automated Electrophoresis Apparatus. Mobility measurements were performed many times and averaged; measurements usually deviated less than $2.5 \%$ from the average.

Dynamic light scattering measurements employed a Coherent mode-stabilized krypton laser operating at a wavelength of $530.9 \mathrm{~nm}$ and a Malvern Instruments spectrometer with multibit correlator. Measured correlation functions were accurately fit by single exponential functions. The reciprocal of the decay time was plotted against the square of the scattering vector; the diffusion coefficient was extracted from the slope of the resulting line. The Stokes-Einstein equation then gave the particles' hydrodynamic radius.

Capillary viscometry measurements were performed with a Ubbelohde capillary viscometer thermostatted at $298.2 \mathrm{~K}$. Typical flow times for the buffer-C12E6 solution were $c a .85 \mathrm{~s}$. Measurements were repeated until the flow times agreed to within $0.1 \mathrm{~s}$.

All other rheological measurements utilized a Bohlin VOR rheometer fitted with one of three geometries: cone-and-plate, single-gap concentric cylinders, and double-gap concentric cylinders for high, medium, and low concentrations. Each sample was loaded into the rheometer and homogenized with shearing at $10 \mathrm{~s}^{-1}$ for $2 \mathrm{~min}$. The first sample, PS2-APEO2, displayed drying problems at high particle concentrations. Drying was hindered by floating a small amount of silicone oil on top of subsequent samples. Excessive shear rates were avoided to prevent any mixing of oil with sample. Samples were recovered for reuse by washing with buffer-C12E6 solution. Low speed centrifugation aliowed removal of dried material and silicone oil. Electrophoresis measurements indicated that the particle mobility was not affected by exposure to the silicone oil. All measurements were completed within a week of the initial grafting reaction.

At constant shear rate, the shear stress and viscosity reached steady-state values within $60 \mathrm{~s}$ of the start of the shear, and reported values reflect averages over 20-60 s. Shear stress and viscosity were reproducible for both increasing and decreasing shear-rate sweeps. Strain sweeps in oscillation (usually at $1 \mathrm{~Hz}$ ) indicated that the storage modulus 


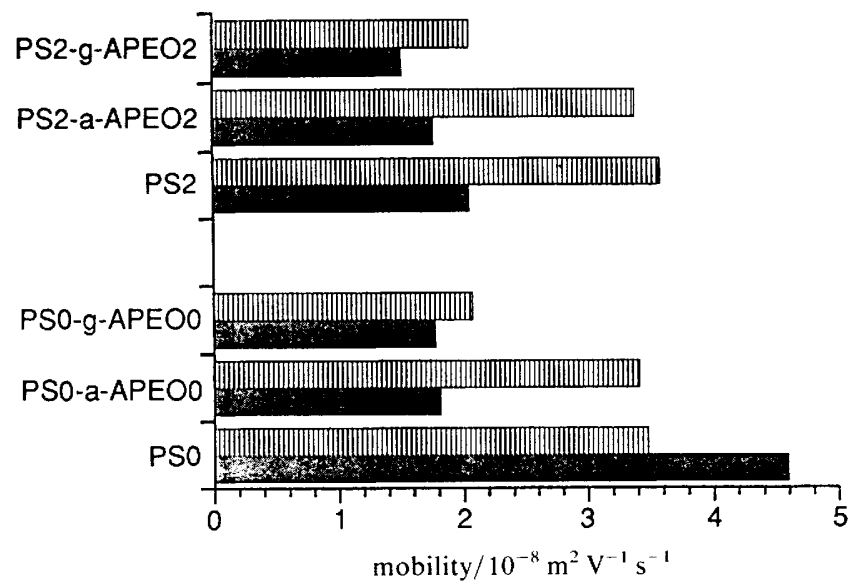

Fig. 1. Electrophoretic mobilities of PS0 and PS2 particles without APEO layers (PSO, PS2), with adsorbed APEO layers (PS0-a-APEO0, PS2-a-APEO2), and with grafted APEO layers (PS0-g-

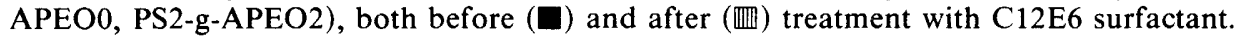

was linear below $1 \%$ strain but strain-softened at higher amplitudes. The loss modulus was linear for strains up to $0.1 \%$; at larger strains, the loss modulus first decreased, then exhibited a broad peak at $c a .5 \%$ strain.

\section{Results and Discussion}

\section{Electrophoresis}

The steady-state velocity of a charged particle in an electric field depends on the balance between electric and viscous forces; the velocity divided by the field strength gives the electrophoretic mobility. Here, mobilities are used for comparison only; interpretation in terms of the electrostatic/polymeric environment around the particles ${ }^{31}$ is not the present concern.

The mobility of PSO particles in $10^{-2} \mathrm{~mol} \mathrm{dm}^{-3}$ buffer varied from ca. $-4 \times$ $10^{-8} \mathrm{~m}^{2} \mathrm{~V}^{-1} \mathrm{~s}^{-1}$ at $\mathrm{pH} 5.0$ to $-6 \times 10^{-8} \mathrm{~m}^{2} \mathrm{~V}^{-1} \mathrm{~s}^{-1}$ at $\mathrm{pH} 8.0$. In the same buffer, PS0 particles covered with adsorbed APEO0 or grafted APEO0 had nearly equal mobilities of $c a .-1 \times 10^{-8} \mathrm{~m}^{2} \mathrm{~V}^{-1} \mathrm{~s}^{-1}$, nearly independent of $\mathrm{pH}$ for values between 5 and 8 . Since the mobility is inversely proportional to the effective particle radius, the hydrodynamic thickness of the APEO layers cannot account for the decrease in the magnitude of the mobility; disturbance of the electrostatic double layer by the APEO must be responsible.

This phenomenon can be used to ascertain whether the APEO is grafted or merely adsorbed. In a displacement experiment, a displacer supplants adsorbed APEO, but grafted APEO remains near the surface. The particle mobilities should reflect this difference. The results of this test are shown in fig. 1; all mobilities were measured in $10^{-3} \mathrm{~mol} \mathrm{dm}^{-3}$ buffer. Samples of bare particles (PS0, PS2), particles with adsorbed APEO (PS0-a-APEO0, PS2-a-APEO2), and particles with grafted APEO (PS0-g-APEO0, PS2-g-APEO2) were treated with excess of C12E6 surfactant. Before treatment, the mobilities of the adsorbed and grafted samples were comparable and relatively low. After treatment, the mobilities of the adsorbed samples rose to roughly that of treated bare particles, while the mobilities of the grafted samples rose slightly but remained low. The former result implies that the adsorbed APEO had been displaced, while the 
Table 1. Dynamic light scattering results

\begin{tabular}{ccccc}
\hline & & \multicolumn{3}{c}{ hydrodynamic layer thickness/nm } \\
\cline { 3 - 5 } PS-APEO & preparation & untreated & added C12E6 & change \\
\hline \multirow{2}{*}{ PS1-APEO2 } & adsorbed & 42 & 35 & -7 \\
PS1-APEO2 & grafted & 54 & 25 & -29 \\
PS2-APEO2 & grafted (273 K) & 86 & 58 & -28 \\
PS2-APEO1 & adsorbed & 9 & 12 & +3 \\
PS2-APEO1 & grafted & 24 & 44 & +20 \\
\hline
\end{tabular}

latter suggests that grafted APEO was indeed grafted. The other possible factor, exposure to the WSC, should have been apparent in the mobilities before surfactant treatment.

In addition, attempts were made to adsorb and to graft APEO0 onto PS0 which had been pretreated with C12E6. In each case, the mobility after exposure to APEO0 equalled the initial mobility of bare PS0. Thus C12E6 prevented both adsorption and grafting of APEO0.

\section{Dynamic Light Scattering}

Measurements of the diffusivities of bare PS1 and PS2 led to hydrodynamic diameters of 119 and $362 \mathrm{~nm}$, respectively; both values are within $5 \%$ of those determined through TEM. The difference between the hydrodynamic radii of APEO-coated and bare particles gave the hydrodynamic thickness of the APEO layer. Table 1 summarizes the results.

For the smaller PS1 particles, grafting of APEO2 produced somewhat thicker layers than adsorption alone; both values are lower than the 95-100 $\mathrm{nm}$ thicknesses for $6.6 \times 10^{5} \mathrm{~g} \mathrm{~mol}^{-1}$ PEO adsorption onto PS reported previously, ${ }^{25,26}$ Grafted APEO2 on the PS2 was thicker than on PS1; perhaps the larger particle size or the different grafting temperature (used for this experiment only) were responsible. Adsorbed APEO1 layers on PS2 were about $50 \%$ thinner than reported values, ${ }^{25,26}$ but the grafted APEO1 layers on PS2 were significantly thicker than the adsorbed layers.

The effect of C12E6 surfactant on the hydrodynamic thickness of the APEO layers is not clear. C12E6 decreased the apparent thickness of APEO2 layers, but the reduction was greater for grafted layers. Layers of APEO1 appeared to increase in thickness after exposure to surfactant. While rationalization of these results is difficult, it is probably safe to say that the C12E6 did not completely displace adsorbed APEO, and that the C12E6 significantly perturbs the configurations of APEO molecules remaining near the PS surface.

\section{Capillary Viscometry}

Saunders' modification of Mooney's equation ${ }^{32}$

$$
\frac{\varphi}{\ln \eta_{\mathrm{r}}}=\frac{1}{k_{1} f}-\frac{\varphi}{k_{1} \varphi_{\mathrm{m}}}
$$

relates the relative viscosity $\eta_{\mathrm{r}}$ to the particle volume fraction $\varphi$ with the Einstein coefficient $k_{1}=2.5$ and $\varphi_{\mathrm{m}}$ as the maximum packing fraction. For each sample, plots of the capillary viscometry data according to eqn (1) were linear. The intercept provided the effective hard-sphere scaling factor, $f$, defined through

$$
f=\frac{\varphi_{\mathrm{e}}}{\varphi}=\left[1+\frac{\delta}{a}\right]^{3}
$$


Table 2. Capillary viscometry results

\begin{tabular}{ccrr}
\hline PS-APEO & $f$ & $a / \delta$ & $\delta / \mathrm{nm}$ \\
\hline PS1-APEO1 & 1.206 & 15.5 & 3.7 \\
PS1-APEO2 & 1.805 & 4.6 & 12.5 \\
PS2-APEO1 & 1.118 & 26.3 & 6.6 \\
PS2-APEO2 & 1.397 & 8.5 & 20.5 \\
\hline
\end{tabular}

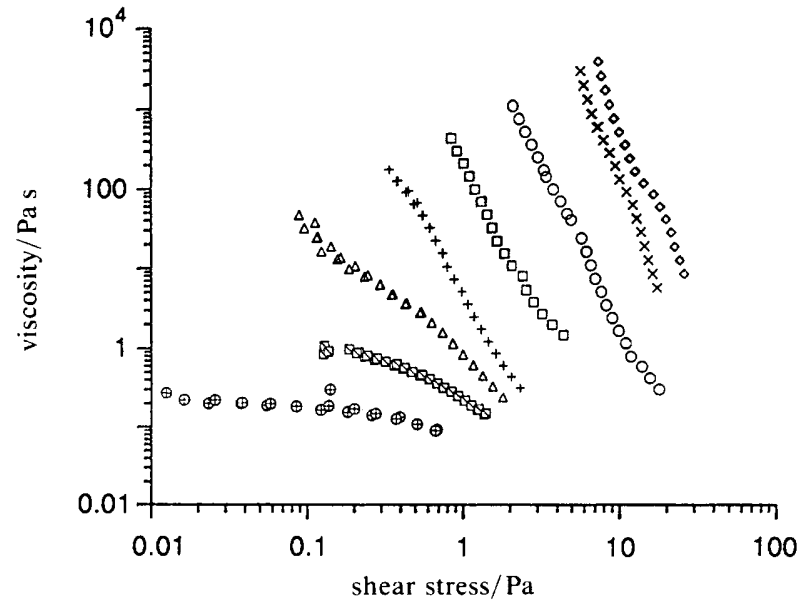

Fig. 2. Viscosity of the PS1-APEO2 suspension as a function of shear stress and particle volume fraction. Particle volume fraction: $\oplus, 0.254 ; \triangle, 0.269 ; \triangle, 0.283 ;+, 0.295 ; \square, 0.348 ; \bigcirc, 0.369$; $\times, 0.400 ; \diamond, 0.433$.

where $\varphi_{\mathrm{e}}$ is the effective hard-sphere volume fraction of the particle plus polymer layer, $\delta$ is the layer thickness, and $a$ is the particle radius. These results are presented in table 2 .

Large values of $f$ or small values of $a / \delta$ indicate 'softness' in the interparticle force, that is less steepness in the force as a function of separation. For a particular particle size, softness increased with the molecular weight of the grafted polymer; for the same grafted polymer, softness decreased with particle size. The capillary viscometry data represent high shear, low $\varphi$ limiting values of the hydrodynamic layer thickness, in contrast to the low-shear, low $\varphi$ values provided by DLS.

\section{Steady-shear Viscometry}

For the four systems listed in table 2, the steady-shear viscosity was found to be a power-law function of shear rate at each particle volume fraction. Fig. 2 shows the viscosity of PS1-APEO2 as a function of shear stress and volume fraction. The general features of the data are typical for all of the systems studied. At low $\phi$, the viscosity reached limiting values at low stress but displayed shear thinning at higher stresses. Viscosities of suspensions with low volume fractions of larger PS2 particles approached limiting values at the highest measured stresses. At all stresses, viscosity increased with $\phi$. Above a critical volume fraction ( $\phi \approx 0.28$ for PS1 suspensions, $\sigma \approx 0.37$ for PS2 suspensions), the viscosity curves exhibited apparent yield stresses. PS2 suspension were iridescent (at rest) for values of $\phi$ above the transition value. 


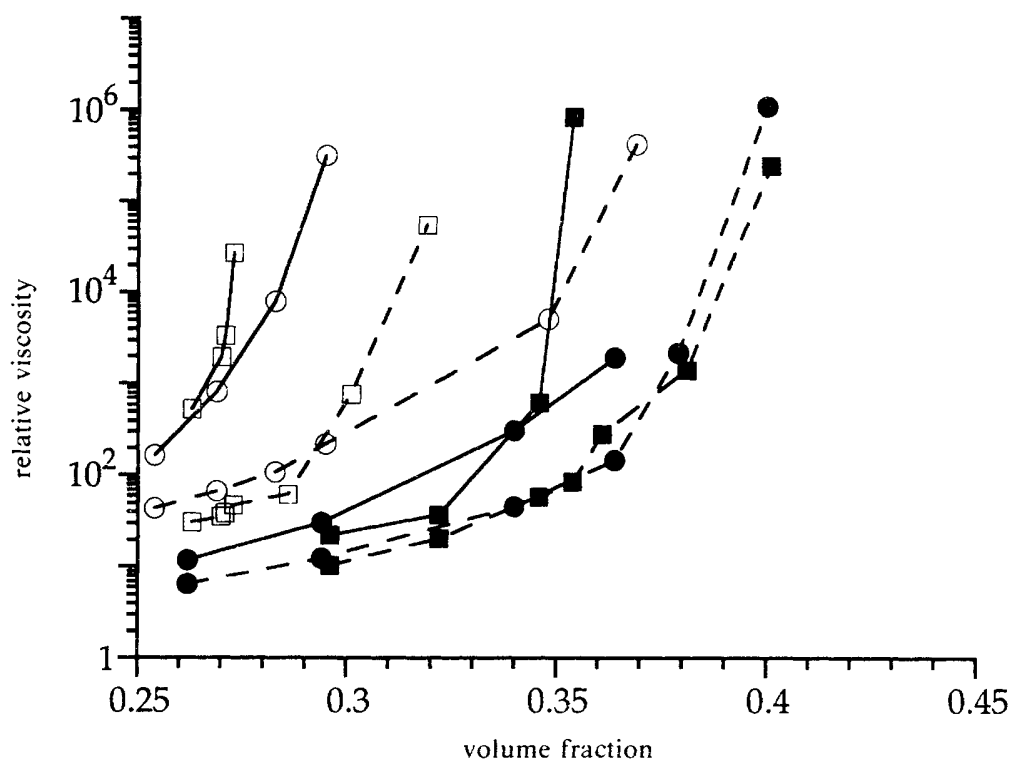

Fig. 3. Relative viscosities of PS-APEO suspensions as functions of volume fraction at two relative stresses: solid curves, $\sigma_{\mathrm{r}}=0.0127$; dashed curves, $\sigma_{\mathrm{r}}=0.127$. $\square$, PS1-APEO1; O, PS1-APEO2; $\square$, PS2-APEO1;, , PS2-APEO2.

The changes in the steady shear rheology with increasing $\phi$ characterize a transition from 'liquid-like' to 'solid-like' behaviour as the mean particle separation becomes comparable to the characteristic length of the interparticle force. The mean particle separation, $h$, scales as

$$
h \approx 2 a\left[\left(\frac{\phi_{\mathrm{m}}}{\phi}\right)^{1 / 3}-1\right] .
$$

Thus $h \approx 35-40 \mathrm{~nm}$ and $h \approx 65-70 \mathrm{~nm}$ for the PS1 and PS2 suspensions, respectively, near the transition values of $\phi$. At low shear rates, the microstructure depends on the balance between Brownian and interparticle forces: Brownian motion randomizes the microstructure of weakly interacting particles, giving liquid-like behaviour, but strong interactions promote short-range order and hinder particle self-diffusion, producing solid-like behaviour. Since the PS1 particles have a much higher diffusivity than the PS2 particles, the interparticle force must be greater in PS1 suspensions than in PS2 suspensions at the transition value of $\phi$. Thus the mean separation at the transition should be smaller in the former. However, the liquid-solid transition is not sharp, and characterization of any true yield stress is problematic, so it is difficult to distinguish the difference between APEO1 and APEO2 layer interactions in this way.

Data at larger stresses provide a better comparison of polymeric interparticle forces. Fig. 3 shows the relative viscosity of each system as a function of volume fraction for two values of the relative stress defined by $\sigma_{\mathrm{r}}=\sigma a^{3} / k_{\mathrm{B}} T$ ( $\sigma$ is the dimensional shear stress). If the particles were Brownian hard spheres, dimensional analysis ${ }^{12}$ indicates that the four curves for each value of the relative stress (solid curves for $\sigma_{\mathrm{r}}=0.0127$, dashed curves for $\sigma_{\mathrm{r}}=0.127$ ) should superimpose. Deviations from this limit reflect details of the interparticle force.

At each relative stress, the viscosities of suspensions of smaller PS1 particles (open symbols) were greater than those of suspensions of larger PS2 particles at the same 
volume fraction. Eqn (3) shows that the mean separation of PS1 particles was about one-third of that of PS2 particles at the same $\phi$. If PS1 and PS2 particles had forceseparation curves with similar magnitudes, then the smaller mean separation of PS1 particles implies stronger interactions, thereby yielding the greater viscosities. Focusing on the solid curves for $\sigma_{\mathrm{r}}=0.0127$, PS1-APEO1 suspensions (open squares) had higher viscosities than PS1-APEO2 suspensions (open circles), suggesting that the force between APEO1 layers was greater than that between APEO2 layers at the same separation. For PS2 particles, the viscosities of APEO1 stabilized suspensions (solid squares) became greater than that of APEO2 stabilized suspensions (solid circles) only for $\phi>0.34$. Comparison of the pairs of curves for PS1 and PS2 imply that the interparticle force differs between small and large particles for at least one of the APEO coatings.

Consider the dashed curves in fig. 3 for the higher relative stress, $\sigma_{\mathrm{r}}=0.127$. Suspensions of larger PS2 particles (solid symbols) stabilized by APEO1 and APEO2 had comparable viscosities at all volume fractions. In this case, domination of the hydrodynamic force over the interparticle force may have rendered the viscosity less sensitive to the latter. If the polymeric forces were greater between the smaller PS1 particles for a given $\phi$, then at the same relative stress, the hydrodynamic force would be less important, and so the viscosity curves might vary with the polymeric force. This appears to be the case, because the viscosities of PS1-APEO1 suspensions became greater than those of the PS1-APEO2 suspensions as $\phi$ increased. Notice the qualitative similarity between the shapes of the PS1 viscosity curves at $\sigma_{\mathrm{r}}=0.127$ and those for PS2 at $\sigma_{\mathrm{r}}=0.0127$ (solid symbols, solid curves). The 'crossover' occurred at a low stress for PS2 suspensions, but at higher stress for PS1 suspensions.

In the limit of high shear stress, the hydrodynamic force dominates the polymeric force, and the polymer layers are significantly distorted from their equilibrium configurations. Capillary viscometry provides data in this limit (table 2). Plotting the data according to eqn (1) identifies the effective hard-sphere radius $a+\delta$ and the scaling factor $f$ [eqn (2)]. In principle, the relative viscosity of an effective hard-sphere suspension is only a function of the effective volume fraction $\phi_{\mathrm{e}}=f \phi$ and the effective relative stress $\sigma_{\mathrm{r}, \mathrm{e}}=f \sigma_{\mathrm{r}}$. The relative viscosities of the four suspensions considered here should be identical functions of $\phi_{\mathrm{e}}$ at the same $\sigma_{\mathrm{r}, \mathrm{e}}$, provided we neglect the variation of the polymeric force with $\sigma_{\mathrm{r}, \mathrm{e}}$. Deviations from effective hard-sphere behaviour then indicate deformation of the APEO layers by hydrodynamic forces. Assuming that the APEO layers suffered the maximum deformation in the capillary viscometer flow, the steady-shear viscosity curves would superimpose only at high values of $\sigma_{\mathrm{r}, \mathrm{e}}$.

In earlier work, ${ }^{10,13,16}$ the Krieger-Dougherty equation ${ }^{12}$

$$
\eta_{\mathrm{r}}=\left[1-\frac{\phi_{\mathrm{e}}}{\phi_{\mathrm{m}}}\right]^{-k_{1} \phi_{\mathrm{m}}}
$$

has successfully fit relative viscosity data for $\phi_{\mathrm{e}}<0.55$. Using the value $\phi_{\mathrm{m}}=0.71$ as indicated for hard spheres in the high-shear limit, ${ }^{10}$ this function is plotted in fig. 4. The experimental data, at constant but much lower $\sigma_{\mathrm{r}, \mathrm{e}}$ had higher relative viscosities as a consequence of shear thinning.

The solid curves in fig. 4 are for $\sigma_{\mathrm{r}, \mathrm{e}}=0.0142$. Unlike the scaling in fig. 3 , which brought curves for the same particle size into proximity, the effective hard-sphere scaling in fig. 4 brings curves for the same APEO molecular weight into proximity. The viscosity curves for APEO1-stabilized suspensions (squares) have the same general shape but do not superimpose. However, curves for APEO2-stabilized suspensions nearly coincide. The better agreement of the viscosities of PS1-APEO2 and PS2-APEO2 suspensions suggests that these were closer to effective hard-sphere suspensions than those stabilized by APEO1. Two explanations are possible. First, the hydrodynamic force could have been stronger than the polymeric force between APEO2 layers, thus making the particle more like effective hard spheres. However, the lack of superposition of the curves in 


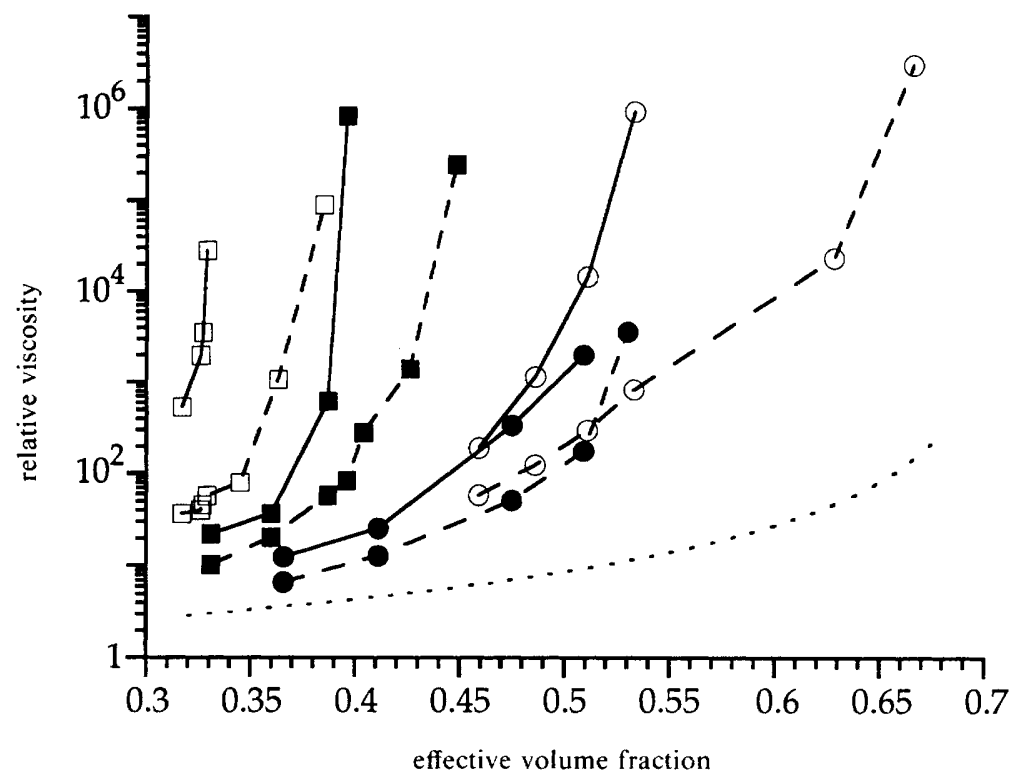

Fig. 4. Relative viscosities of PS-APEO suspensions as functions of effective volume fraction at two effective relative stresses: solid curves, $\sigma_{\mathrm{r}}=0.0142$; dashed curves, $\sigma_{\mathrm{r}}=0.142$. $\square$, PS1-APEO1; O, PS1-APEO2; $\square$, PS2-APEO1; - PS2-APEO2. The dotted curve is Krieger's equation [eqn (4)] with $\phi_{\mathrm{m}}=0.71$.

fig. 3 for $\sigma_{\mathrm{r}}=0.0127$ (differing from $\sigma_{\mathrm{r}, \mathrm{e}}$ only by the factor $f$ ) implies that hydrodynamic forces were not dominant.

The second possible explanation is that APEO1 and APEO2 layers exhibited different degrees of compression at the same effective stress; the reasoning here is based on the presumption that the effective hard-sphere scaling used in fig. 4 is associated with maximum layer compression at high stresses. Relative to this limit, the better superposition of the viscosity curves of APEO2 suspensions suggests that APEO2 layers were more deformed (compressed) than APEO1 layers at the same $\sigma_{\mathrm{r}, \mathrm{e}}$. Effective hard-sphere scaling seems to improve at a higher stress, $\sigma_{\mathrm{r}, \mathrm{e}}=0.142$ (dashed curves in fig. 4): the viscosities of APEO2-stabilized suspensions (circles) agreed over a larger range of $\phi_{\mathrm{e}}$, and the viscosity curves for APEO1-stabilized suspensions (squares) moved closer together.

\section{Oscillatory Measurements}

Fig. 5 depicts the storage modulus of the PS1-APEO2 suspension as a function of frequency $(\omega)$ and volume fraction. The corresponding plots for the other suspensions had the same qualitative features. At low $\phi$, the particles were well separated and the polymeric interactions were weak; thus the storage modulus was relatively small. At low frequency, the characteristic time for rearrangement of the suspension microstructure was much less than that of the oscillatory deformation, so the suspensions dissipated energy through particle diffusion rather than storing energy in polymeric interactions. At high frequency, though, the oscillation was faster than some of the diffusive modes of dissipation, so the storage modulus increased with frequency. Such behaviour characterizes liquid-like particle microstructure; however, the slope of the $\log \left(G^{\prime}\right)$ $\log (\omega)$ plot was not 2 as expected for a true viscoelastic liquid. Even at volume fractions as low as 0.25 , there must have been significant polymeric interactions between particles. 


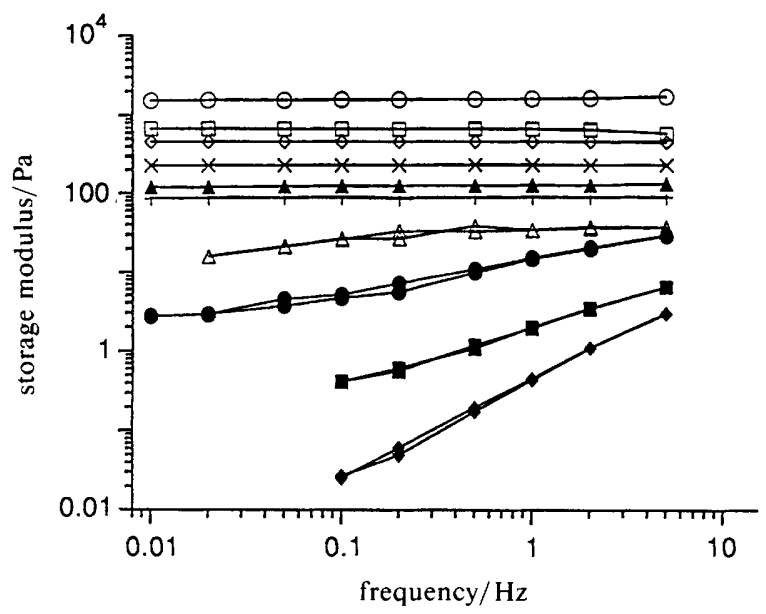

Fig. 5. Storage modulus of the PS1-APEO2 suspension as a function of oscillation frequency and particle volume fraction. Particle volume fraction: $\bullet, 0.254 ; \square, 0.269 ; 0,0.283 ; \triangle, 0.295$; ,$+ 0.318 ; \Delta, 0.348 ; \times, 0.369 ; \diamond, 0.400 ; \square, 0.433 ; 0,0.496$.

The storage modulus increased with volume fraction as interactions became stronger and more numerous. The increased interactions hindered particle self-diffusion so that the spectrum of timescales for microstructural rearrangement shifted to longer times. The behaviour at high $\phi$ was typical of a viscoelastic solid: the oscillation time was much faster than all diffusive relaxation processes, leaving the storage modulus independent of frequency. The transition from liquid-like to solid-like viscoelastic behaviour occurred at about the same critical volume fraction as was observed in the viscosity-shear stress results in fig. 2. This agreement was found for each suspension.

Dimensionless scaling of oscillatory data ${ }^{13}$ defines the relative storage modulus $G^{\prime} a^{3} / k_{\mathrm{B}} T$ and the relative frequency $\omega a^{3} \eta_{\mathrm{m}} / k_{\mathrm{B}} T$ with $\eta_{\mathrm{m}}$ as the viscosity of the medium. The relative storage modulus of Brownian hard spheres thus depends only on the relative frequency and the volume fraction. Fig. 6 shows the relative storage modulus as a function of volume fraction for all of the suspensions at a constant relative frequency of $4.15 \times 10^{-5}$. The relative storage modulus of each suspension rose steeply at about the same volume fraction found for the viscoelastic liquid-solid transition in the steady shear data. For $\phi<0.40$, the curves clearly do not superimpose, presumably due to differing polymeric interactions between particles. The relative moduli of suspensions of smaller PS1 particles were greater than those of larger PS2 particles. Again, this is a consequence of the smaller mean separations and stronger interactions of PS1 particles compared to PS2 particles at the same $\phi$. For each particle size, the relative modulus of APEO1-stabilized suspensions (squares) was greater than that for those stabilized by APEO2 (circles). This observation implies that the force between APEO1 layers was greater than that between APEO2 layers at the same separation. The viscosity data in fig. 3 (solid curves) and the modulus data in fig. 6 are consistent in support of this hypothesis.

For $\phi>0.40$, the relative storage modulus increased roughly exponentially with volume fraction. The moduli of PS1-APEO2 and PS2-APEO2 suspensions were particularly close. As the mean separation became smaller, the particles interacted more strongly, increasing the moduli. If the polymeric force-separation functions in the two suspensions varied in a similar way over that range of separations, then the storage moduli would increase at the same rate. Alternatively, if the polymeric forces were steep enough, then the particles might have been acting as effective hard spheres; in 


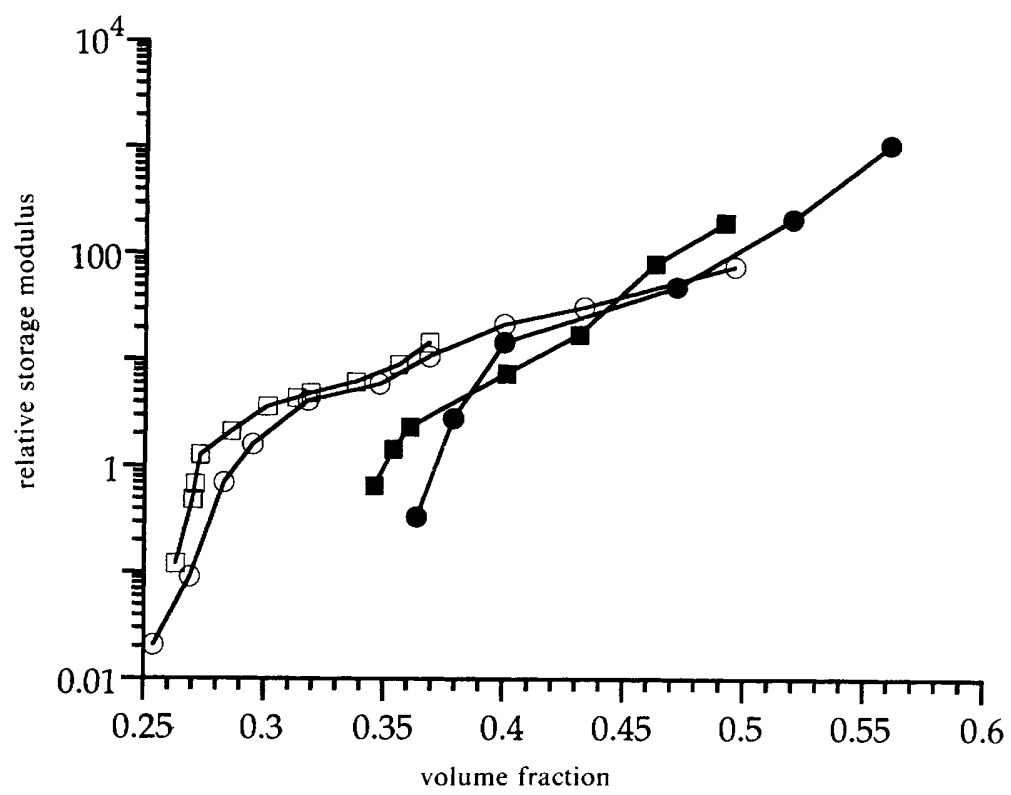

Fig. 6. Relative storage moduli of PS-APEO suspensions as functions of volume fraction at a relative frequency of $4.15 \times 10^{-5}$. $\square$, PS1-APEO1; O, PS1-APEO2; $\mathbf{1}$, PS2-APEO1;, PS2-APEO2.

this case the moduli would depend only weakly on the details of the force-separation functions. The relative storage modulus of the PS2-APEO1 suspension increased more rapidly than the other two suspensions in this volume fraction range. PS2-APEO1 particles were arguably the 'hardest' according to the capillary viscosity data in table 2. These observations can be rationalized if the force between APEO1 layers was a steeper function of separation than that between APEO2 layers.

\section{Conclusions}

The rheological behaviour of PS suspensions stabilized by high molecular weight grafted APEO depends intimately upon the details of the grafting process, the resultant polymer layer structure, and the variation of the polymeric interparticle force with particle separation. The same can be said for suspensions stabilized by low molecular weight species, such as silica-octadecyl alcohol ${ }^{9,10}$ or PMMA-poly(hydroxystearic acid), ${ }^{13}$ but the contrast between these 'effective hard sphere' suspensions and those studied here is noteworthy. The differences arise ultimately from the nature of the grafting process. Low molecular weight stabilizers usually are not adsorbed onto the particle substrate; instead, grafting is accomplished through extreme reaction conditions, ${ }^{10}$ incorporation of the stabilizer during particle polymerization, ${ }^{13}$ or by strong adsorption of an insoluble block of a block copolymer. ${ }^{16}$ Effective stabilization is achieved by maximizing the graft density, thereby forming a thin but dense stabilizer layer with minimum compressibility. These characteristics contribute to the success of effective hard-sphere scaling in rationalizing the rheological behaviour.

High molecular weight APEO, on the other hand, is readily adsorbed onto PS particles. The graft density is probably controlled by the adsorption behaviour; although reasonable, this conjecture has not been tested experimentally. The electrophoresis and DLS data given above imply that grafted and adsorbed APEO layers have similar structure, at least before treatment with C12E6 surfactant. Many studies ${ }^{1,2,4}$ have shown that such polymer layers are diffuse and have density profiles that gradually decay with 
increasing distance from the particle surface. Two distinct implications for suspension rheology should be considered: the polymeric force gradually increases in magnitude over a long range as particle separation decreases; and the extended layers may be more susceptible to deformation by hydrodynamic forces. Certainly, at least the first effect is manifested in the rheological data presented in fig. 2-6.

Relating the rheological behaviour to the details of the APEO layer structures is the prime objective, but the task is complicated by several factors. The main deficiency in the present study is the lack of data on grafted or adsorbed amounts of APEO per unit area of PS particle surface. Modelling studies ${ }^{4}$ have shown that adsorbed amount is the key factor linking polymer layer structure to polymeric forces. Independent measurement of adsorbed/grafted amounts would help clarify the details of the grafting mechanism (for example, the dependence of grafting efficiency on adsorbed amount) as well as assist in rationalizing the rheology. In particular, some of the differences among the viscosity curves in fig. 3 for a given APEO could be attributed to different graft densities. PS1 was prepared without surfactant, while the PS2 polymerization included dodecanoic acid. Dialysis probably did not completely remove the surfactant from the PS2. If APEO adsorption and grafting were hindered by the surfactant, then the polymeric force might be less between large particles than between small particles for the same APEO stabilizer.

Two other factors complicate the interpretation of the data presented here and merit further study. First, the behaviour of the suspensions during centrifugation and redispersion, and the electrophoresis and DLS measurements, indicated that the C12E6 surfactant had a profound effect on the properties of the APEO-stabilized suspensions. The C12E6 could have associated with the APEO through a group-specific chemical interaction such as hydrogen bonding, or a less-specific physical interaction may have been responsible. Any further analysis of the macroscopic properties of the suspension that relies upon models of polymer layer structure must take the surfactant into account. A better understanding of such polymer-surfactant interactions would have an impact upon the performance of many materials, including paints, inks, oil recovery fluids, and consumer products.

Secondly, electrostatic effects were generally ignored. This was probably a good approximation considering the low mobility of the coated particles and the ionic strength of the buffer. Even so, there may have been some dependence of APEO adsorption, grafting efficiency, or layer structure on the electrostatic environment around the PS particles. Coupling between the polymer layer and electrostatic double-layer structures becomes more important at very high and very low ionic strengths: in the former case, the finite volume occupied by the ions perturbs the polymer configurations, while in the latter, the electrostatic force has a range comparable to or greater than the polymeric force. Indeed, the polymeric and electrostatic components of the total interparticle force are more likely to be coupled than separable and additive. These considerations are vital for technologies involving interactions mediated by polyelectrolytes, enzymes, proteins, or other biological materials.

H.J.P. gratefully acknowledges the financial support of the U.S. National Science Foundation under grant INT-8806327, and thanks all at the University of Bristol for their hospitality during his stay there. H.J.P. and J.W.G. thank Mr John Dimery and Dr Keith Ryan for their technical support and advice.

\section{References}

1 D. H. Napper, Polymeric Stabilization of Colloidal Dispersions (Academic, New York, 1983).

2 T. Sato and R. Ruch, Stabilization of Colloidal Dispersions by Polymer Adsorption (Marcel Dekker, New York, 1980).

3 Flocculation and Dewatering, ed. B. M. Moudgil and B. J. Scheiner (AIChE, New York, 1988). 
4 H. J. Ploehn and W. B. Russel, Adv. Chem. Eng., 1990, 15, 137.

5 Dispersion Polymerization in Organic Media, ed. K. E. J. Barnett (Wiley, New York, 1975).

6 R. J. R. Cairns, R. H. Ottewill, D. W. J. Osmond and I. Wagstaff, J. Colloid Interface Sci., 1976, 54, 45.

7 A. K. van Helden, J. W. Jansen and A. Vrij, J. Colloid Interface Sci., 1981, 81, 354.

8 K. Ryan, Chem. Ind., 6 June 1988.

9 A. Vrij, J. W. Jansen, J. K. G. Dhont, C. Pathmamanoharan, M. M. KopsWerkhoven and H. M. Fijnaut, Faraday Discuss. Chem. Soc., 1983, 76, 19.

10 C. G. de Kruif, E. M. F. van Iersel, A. Vrij and W. B. Russell, J. Chem. Phys., 1985, 83, 4717; J. Mellema, C. G. de Kruif, C. Blom and A. Vrij, Rheol. Acta, 1987, 26, 40; J. C. van der Werff and C. G. de Kruif, J. Rheol., 1989, 33, 421; J. C. van der Werff, C. G. de Kruif and J. K. G. Dhont, Physica A, 1989, 160, 205; J. C. van der Werff, C. G. de Kruif, C. Blom and J. Mellema, Phys. Rev. A, $198939,795$.

11 G. K. Batchelor, J. Fluid Mech., 1970, 41, 545; G. K. Batchelor and J. T. Green, J. Fluid Mech., 1972, 56, 401; G. K. Batchelor, J. Fluid Mech., 1977, 83, 97.

12. I. M. Krieger, Trans. Soc. Rheol., 1963, 7, 101; I. M. Krieger, Adv. Colloid Interface Sci., 1972, 3, 111.

13 W. J. Frith, PhD Dissertation (Catholic University, Leuven, Belgium, 1986); W. J. Frith, J. Mewis and T. A. Strivens, Powder Technol., 1987, 51, 27; J. Mewis, W. J. Frith, T. A. Strivens and W. B. Russel, AIChE J., 1989, 35, 415.

14 T. A. Strivens, Colloid Polym. Sci., 1983, 261, 74; T. A. Strivens, Colloid Polym. Sci., 1987, $265,553$.

15 S. J. Willey and C. W. Macosko, J. Rheol., 1978, 22, 525.

16 G. N. Choi and I. M. Krieger, J. Colloid Interface Sci., 1986, 113, 94; 113, 101.

17 M. D. Croucher and T. H. Milkie, in The Effect of Polymers on Dispersion Properties, ed. Th. F. Tadros (Academic Press, New York, 1982), p. 101.

18 M. C. A. Griffin, J. C. Price and W. C. Griffin, J. Colloid Interface Sci., 1989, 128, 223.

19 M. D. Croucher and T. H. Milkie, Faraday Discuss. Chem. Soc., 1983, 76, 261.

20 T. Milkie, K. Lok and M. D. Croucher, Colloid Polym. Sci., 1982, 260, 531.

21 C. Prestidge and Th. F. Tadros, J. Colloid Interface Sci., 1988, 124, 660.

22 M. A. Ansarifar and P. F. Luckham, Colloid Polym. Sci., 1982, 267, 736.

23 Th. F. Tadros, ACS Symp. Ser., 1984, 240, 411.

24 U.S. patent, Seragen, Inc.

25 T. Kato, K. Nakamura, M. Kawaguchi and A. Takahashi, Polym. J., 1981, 13, 1037; C. Cowell and B. Vincent, in The Effect of Polymers on Dispersion Properties, ed. Th. F. Tadros (Academic Press, New York, 1982), p. 263; J. A. Baker and J. C. Berg, Langmuir, 1988, 4, 1055.

26 M. A. Cohen Stuart, F. H. W. H. Waajen, T. Cosgrove, B. Vincent and T. L. Crowley, Macromolecules, $1984,17,1825$.

27 J. W. Vanderhoff, H. J. van den Hul, R. J. M. Tausk and J. Th. G. Overbeek, in Clean Surfaces: Their Preparation and Characterization, ed. G. Goldfinger (Dekker, New York, 1970), p. 15.

28 J. W. Goodwin, R. H. Ottewill, R. Pelton, G. Vianello and D. E. Yates, Br. Polym. J., 1978, $10,173$.

29 J. March, Advanced Organic Chemistry (Wiley-Interscience, New York, 1985), p. 350, 372.

30 S. J. Partridge, Ph.D Dissertation (University of Bristol, 1987).

31 K. L. Koopal, V. Hlady and J. Lyklema, J. Colloid Interface Sci., 1988, 121, 49.

32 F. L. Saunders, J. Colloid Sci., 1961, 16, 13. 